\title{
SERUM HOMOCYSTEINE AND HIGH-SENSITIVE C-REACTIVE PROTEIN LEVELS AND CARDIOVASCULAR DISEASE IN PATIENTS WITH DIABETES MELLITUS TYPE 2
}

\author{
Yana Bocheva, Rumyana Pateva, Rumyana Ilieva \\ Central Clinical Laboratory, St. Marina University Hospital of Varna, \\ Medical University of Varna
}

\begin{abstract}
PURPOSE: Hyperhomocysteinaemia and hs-CRP are non-classical risk factors independently associated to the development of cardiovascular disease. The increased coronary heart disease (CHD) risk in subjects with type 2 diabetes mellitus (DMT2) can be in part explained by the classical risk factors like hypertension, hyperlipidemia and obesity. There is no sufficient data about the importance of homocysteine (Hcy) and hsCRP levels in DMT2 patients without CHD in predicting the macroangiopathic complications. The aim of this study was to determine the association between plasma homocysteine and hs-CRP concentrations in DMT2 patients with and without CHD.

MATERIAL AND METHODS: Fifty patients hospitalized in the Clinic of Endocrinology at St. Marina University Hospital of Varna were divided into three groups: group one, 20 DMT2 patients without any evidence of CHD; group two, 20 DMT2 patients with history of CHD and group three, 10 healthy controls matched to these groups in terms of age and classical risk factors for CHD.

RESULTS: Serum hs-CRP concentrations were significantly higher in DMT2 patients than in healthy controls as well as in DMT2 patients with CHD than in CHD-free DMT2 ones. Serum Hcy concentrations were significantly higher in DMT2 patient with CHD than in controls. Both markers were elevated in group two presenting with the highest risk for CHD.
\end{abstract}

Key words: homocysteine, hs-CRP, type 2 diabetes mellitus, coronary heart disease, risk factors

\section{INTRODUCTION}

The prevalence of cardiovascular disease (CVD) in patients with diabetes mellitus (DM) is by two-four times higher and represents the leading cause for death in them. Classical CVD risk factors such as tobacco smoking, hypertension, hypercholesterolaemia and obesity are not sufficient to explain this increasing

Address for correspondence:

Yana Bocheva, $M D$

Central Clinical Laboratory,

St. Marina University Hospital of Varna,

1 Hristo Smirnenski Str.,

9010 Varna, Bulgaria

E-mail:y_bocheva@yahoo.com

Received: August 30, 2012

Accepted: January 15, 2013 risk for DM patients anymore (11). Researchers are focusing on investigating new risk factor such as factors of the oxidative stress, free radicals, some metabolites and hormones like testosterone. Hyperhomocysteinaemia and hs-CRP are nonclassical risk factors independently associated to CVD development. Althouigh the importance of the acute phase protein in its low concentrations known as hs$\mathrm{CPR}$ is proved in many studies, data about the role of homocysteine (Hcy) in predicting CHD remains controversial, especially in specific groups of patient like those with type $2 \mathrm{DM}$ (DMT2).

The therapeutic possibilities for lowering serum Hcy levels are well-known and very attractive to scientists. One still looks for the answer of the question whether hyperhomocysteinaemia is the cause or the effect of ongoing atherogenesis. 
Hcy is a sulphur-containing amino acid in the body produced by conversion of methionine, an essential amino acid present in foods regularly consumed within the diet. Low Hcy levels (5-15 $\mu \mathrm{mol} / \mathrm{L}$ ) are normally found in plasma (5). However, genetic defects in the enzymes of Hcy metabolism markedly increase Hcy levels. Studies in animal models show that elevated Hcy levels result in increased oxidative stress $(3,9)$, impaired endothelial function $(10,12)$ and increased thrombogenicity $(10)$, which act together to promote atherosclerosis (4).

Some investigations demonstrate that plasma Hcy levels are elevated in CVD patients with and without DM. However, the results dealing with the relationship between plasma Hcy levels and CVD in DMT2 patients are controversial. Some studies report slightly elevated Hcy levels in DMT2 patients with CHD and low or normal levels in DMT1 ones, which leads to the conclusion that Hcy levels do not influence on CHD prevalence in DM patients $(2,8)$. Other investigations prove that hyperhomocysteineaemia is an independent risk factor for CHD in DMT2 patients when compared to those without DM $(7,8)$.

The aim of this study was to determine the association between plasma homocysteine and hsCRP concentrations in DMT2 patients with and without CHD.

\section{MATERIAL AND METHODS}

After approval of the study protocol by the local ethical committee, the study was conducted in the CentralClinicalLaboratoryandClinic ofEndocrinology at St. Marina University Hospital of Varna. Inclusion criterion was diagnosis of DMT2 according to World Health Organization (WHO) criteria for at least 2 years. Exclusion criteria were previously recorded myocardial infarction, angina pectoris, stroke, coronary artery by-pass surgery, percutaneous transluminal coronary angioplasty, uncontrollable arterial hypertension (blood pressure $\geq 180 / 100 \mathrm{~mm} \mathrm{Hg}$ ), medications like digitalis or drugs affecting homocysteine metabolism (including vitamins), peripheral vascular disease, tobacco smoking, alcohol consumption, diagnosis of hepatic and/or thyroid disease according to physical examination and laboratory findings, arrhythmic or ischemic changes in electrocardiogram (ECG) at rest, severe acute or chronic disease, serum creatinine $>140 \mathrm{mmol} / \mathrm{L}$, and presence of retinoangiopathy or nephropathy. Age- and sex-matched healthy subjects were chosen as controls. Their medical histories, physical examinations, routine biochemical and hematological tests and ECG records were in normal ranges. They were not on any medication (including vitamins) and did not report any tobacco or alcohol abuse.

The study covered 50 patients in order to investigate the association between plasma CRP and Hcy levels, the presence of CHD and incidence of cardiovascular events among DMT2 patients. The first group consisted of 20 patients with DMT2, 12 females and 8 males. All of them did not present with any clinical, laboratory or instrumental evidence of $\mathrm{CHD}$ or CVD. The second group consisted of 20 patients, 10 males and 10 males, with DMT2 and a history of CHD or CVD. The third control group consisted of 10 subjects, 5 males and 5 females, matched to these groups in terms of age and classical risk factors for CHD. Every participant in the study signed an informed agreement that was approved by the local ethical committee.

The laboratory constellation included serum high sensitive CRP (hs-CRP), serum cholesterol, HDL-cholesterol, triglycerides, LDL-cholesterol and serum Hcy levels.

Venous blood samples were taken from the patients after 12-hour fasting. Total cholesterol and triglyceride levels were measured by using standard commercial enzymatic kits. LDL-cholesterol concentration was calculated by Friedewald's formula. Plasma obtained by addition of EDTA and centrifugated immediately after collection was used for Hcy estimation. Plasma samples were frozen and stored at $-20^{\circ} \mathrm{C}$. Hcy levels were determined by polarization immunoassay method using Abbott IMX analyser. Hs-CRP was determined by using Pointe scientific test adapted on Hitachi 902.

\section{RESULTS}

The laboratory results of the patients and controls were shown on Table 1. There were no statistically significant differences in the biochemical 
Serum homocysteine and high-sensitive C-reactive protein levels and cardiovascular disease ...

Table 1. Laboratory characteristics of patients and controls

\begin{tabular}{l|c|c|c|}
\hline Parameter & Group one & Group two & Group three \\
\hline Age (years) & $54 \pm 6$ & $58 \pm 2$ & $50 \pm 4$ \\
Body mass idex $\left(\mathrm{kg} / \mathrm{m}^{2}\right)$ & $30 \pm 2$ & $31 \pm 3$ & $28 \pm 3$ \\
Fasting plasma glucose $(\mathrm{mmol} / \mathrm{L})$ & 8,1 & 8,8 & 5,2 \\
Total cholesterol $(\mathrm{mmol} / \mathrm{L})$ & 6,8 & 7,2 & 6,5 \\
Triglycerides $(\mathrm{mmol} / \mathrm{L})$ & 2,7 & 3,1 & 2,6 \\
High density cholesterol $(\mathrm{mmol} / \mathrm{L})$ & 1,1 & 1,0 & 1,3 \\
Low density cholesterol $(\mathrm{mmol} / \mathrm{L})$ & 3,6 & 3,7 & 2,4 \\
hs-CRP $(\mathrm{mg} / \mathrm{L})$ & 3,77 & 5,8 & 2,18 \\
Hcy $(\mu \mathrm{mol} / \mathrm{L})$ & 8,9 & 12,66 & 8,1 \\
\hline
\end{tabular}

characteristics, age and body mass index (BMI) of the participants except forr the fasting plasma glucose concentrations.

Gender distribution of Hcy and hs-CRP values in patients and controls were presented on Table 2. atherosclerosis. There are a lot of studies indicating the role of Hcy as a non-classical independent risk factor for CHD, however, information about DM patients remains controversial. Mc Cully was the first to draw attention to the relationship between Hcy

Table 2. Gender distribution of Hcy and hs-CRP values in patients and controls

\begin{tabular}{|c|c|c|c|c|c|c|}
\hline \multirow{2}{*}{ Parameter } & \multicolumn{2}{|c|}{ Group one } & \multicolumn{2}{|c|}{ Group two } & \multicolumn{2}{|c|}{ Group three } \\
\hline & females & males & females & males & females & males \\
\hline hs-CRP (mg/L) & 3,03 & 4,2 & 4,97 & 5,95 & 2,1 & 2,3 \\
\hline $\mathrm{Hcy}(\mu \mathrm{mol} / \mathrm{L})$ & 10,8 & 8,2 & 12,41 & 9,2 & 8,95 & 7,8 \\
\hline
\end{tabular}

According to these results, it was evident that there was a significant difference between the hs-CRP concentration $(\mathrm{p}<0,001)$ of DMT2 2 atients and controls as well as between those of group one and group two. This was, however, not the case of Hcy. Although Hcy concentrations of DMT2 patients were higher than those of controls, there was no any statistically significant difference $(\mathrm{p}=0,2)$. On the other hand, Hcy concentrations differed statistically significantly $(p<0,001)$ of between group one and group two.

\section{DISCUSSION}

Elevated plasma Hcy and hs-CRP levels are wellknown as independent CVD risk factors. However, in the absence of a clear mechanism linking Hcy to $\mathrm{CVD}$, there is an ongoing debate whether this relationship is a cause or a result of the ongoing levels and CVD in 1967. This topic has been subject to intensive research, especially, because of the easy way to control hyperhomocysteinemia by vitamin $\mathrm{B}$, folate and nicotinamid acid supplementation.

It has been shown (10) that Hcy levels are higher in patients with established macroangiopathy which leads to the conclusion that Hcy levels are an independent risk factor for CVD in DMT2 patients, too. Other authors report that hyperhomocysteinemia is by 1,6 times stronger CVD risk factor in DM patients than in the common population (8). These data and the fact that CVD prevalence in DM patients is by 2-4 times higher and remains the leading cause for death in this group initiated numerous studies on hyperhocysteinemia, DM and CVD. 
Our study failed to prove that examining Hcy of every DM patient without CHD complication can be useful for CHD risk stratification. It is, however, obvious that Hcy is a part of the portrait of CHDcomplicated DM patient. Unfortunately, it is not possible to give a clear answer to this question with our findings yet. Further prospective long-term outcome studies will help elucidating the issue whether high Hcy levels in DM patients are a primary independent risk factor or an early finding of arteriosclerosis.

\section{CONCLUSION}

Serum hs-CRP concentrations are significantly higher in DMT2 patients than in healthy controls as well as in DMT2 patients with CHD than in those without CHD. Serum Hcy concentrations are significantly higher in DMT2 patients with CHD than in controls. Both markers are elevated in the highest risk group of DMT2 patients and CHD. Our results support the use of hs-CRP as risk stratification tool although this assumption can't be directly addressed to Hcy presenting with a questionable diagnostic value.

\section{REFERENCES}

1. Buysschaert, M., A. Dramois, P. Wallemaco, P. Hermans. Hyperhomocysteinemia in type II diabetes. Relationship to macroangiopathy, nephropathy and insulin resistance.- Diabetes Care, 23, 2000, No 12, 1816-1822.

2. Cronin, C., J. Mc Partlin, F. Barry, F. Borruy, J. Scott, D. Weir. Plasma homocysteine concentrations in type I diabetes.- Diabetes Care, 21, 1998, No 11, 1843-1847.

3. De Chiara, B., V. Sedda, M. Parolini, J. Campolo, R. De Maria, R. Caruso, et al. Plasma total cysteine and cardiovascular risk burden: action and interaction.- Sci. World J., 2012; 303654. doi: $10.1100 / 2012 / 303654$.

4. El-Khairy, P., M. Ueland, H. Refsum, I. M. Graham, S. E. Vollset. Plasma total cysteine as a risk factor for vascular disease: the European concerted action project.- Circulation, 103, 2001, No 21, 2544-2549.
5. Frantzen, F., A. L. Faaren, I. Alfheim, A. K. Nordhei. Enzyme conversion immunoassay for determining total homocysteine in plasma or serum.- Clin. Chem., 44, 1998, No 2, 311-316.

6. Harrison, D., K. K. Griendling, U. Landmesser, B. Hornig, H. Drexler. Role of oxidative stress in atherosclerosis.- Am. J. Cardiol., 91, 2003, No 3, 7A-11A.

7. Hofmann, M. A., E. Lalla, Y. Lu, M. R. Gleason, B. M. Wolf, N. Tanji, et al. Hyperhomocysteinemia enhances vascular inflammation and accelerates atherosclerosis in a murine model.- J. Clin. Invest., 107, 2001, No 6, 675-683.

8. Hoogeveen, E. K., P. J. Kostense, P. J. Beks, A. J. C. Mackooy. Hyperhomocysteinemia is associated with an increased risk of cardiovascular disease, especially in NIDDM. A population-based study.Arterioscl. Thromb. Vasc. Biol., 18, 1998, No 1, 133-138.

9. Kanani, P. M., C. A. Sinkey, R. L. Browning, M. Allaman, H. R. Knapp, W. G. Haynes. Role of oxidant stress in endothelial dysfunction produced by experimental hyperhomocyst(e)inemia in humans.- Circulation, 100, 1999, No 11, 1161-1168.

10. Lentz, S. R. Mechanisms of homocysteine-induced atherothrombosis.- J. Thromb. Haemost., 3, 2005, No 8, 1646-1654.

11. Stabler, S. B., E. Raymond, B. W. Jeffers, J. Cohen, R. Allen, R. W. Schrier. Total homocysteine is associated with nephropathy in non-insulin dependent DM.- Metabolism, 48, 1999, No 9, 1096-1101.

12. Stamler, J. S., J. A. Osborne, O. Jaraki, L. E. Rabbani, M. Mullins, D. Singel, J. Loscalzo. Adverse vascular effects of homocysteine are modulated by endothelium-derived relaxing factor and related oxides of nitrogen.- J. Clin. Invest., 91, 1993, No 1, 308-318. 